\title{
A Remark on the Localisation of a Charged Bose Field
}

\author{
I. F. WILDE*
}

Seminar für Theoretische Physik, ETH, Zürich

Received May 24, 1971

\begin{abstract}
We give two natural definitions for the local field algebras associated with a massive relativistic charged Bose field. These are both covariant, but are relatively antilocal in the sense of Segal and Goodman [1].

They have the same representation of the Poincare group, but are not in the same Borchers class [2].
\end{abstract}

\section{Introduction}

The purpose of this note is to point out that there are two relativistically covariant descriptions of the charged field in terms of local field algebras. These are not essentially the same, as one might expect; rather they are antilocal with respect to each other [1].

We begin by giving the notation.

The Fock space for the charged field is given by $\mathscr{F}=\mathscr{F}_{+} \otimes \mathscr{F}_{-}$ where $\mathscr{F}_{ \pm}$is the usual symmetric Fock space over $L^{2}\left(\mathbb{R}^{3}, d \Omega\right) ; d \Omega$ $=d^{3} k / 2 \sqrt{\left(\boldsymbol{k}^{2}+m^{2}\right)}$. The charged field $\phi(x)$ and its time-derivative, $\pi(x)$, are defined as operator-valued distributions by

$$
\begin{aligned}
& \phi(x)=(2 \pi)^{-3 / 2} \int_{k^{0}=\omega(\boldsymbol{k})}\left[e^{i(k, x)} a_{+}^{*}(\boldsymbol{k}) \otimes \mathbf{1}+\mathbf{1} \otimes a_{-}(\boldsymbol{k}) e^{-i(k, x)}\right] \frac{d^{3} k}{\sqrt{2 \omega(\boldsymbol{k})}} \\
& \pi(x)=i(2 \pi)^{-3 / 2} \int_{k^{0}=\omega(\boldsymbol{k})}\left[e^{i(k, x)} a_{+}^{*}(\boldsymbol{k}) \otimes \mathbf{1}-\mathbf{1} \otimes a_{-}(\boldsymbol{k}) e^{-i(k, x)}\right] \sqrt{\frac{\omega(\boldsymbol{k})}{2}} d^{3} k
\end{aligned}
$$

where $(k, x)=k^{0} x^{0}-\boldsymbol{k} \cdot \boldsymbol{x}, \omega(\boldsymbol{k})=\sqrt{\boldsymbol{k}^{2}+m^{2}}$ and $a_{ \pm}^{\#}(\boldsymbol{k})$ are the annihilation and creation forms on $\mathscr{F}_{ \pm}$:

$$
\begin{gathered}
a_{ \pm}(\boldsymbol{k}): \Psi_{ \pm}\left(\boldsymbol{k}_{1}, \ldots, \boldsymbol{k}_{n}\right) \mapsto \sqrt{\frac{n}{2 \omega(\boldsymbol{k})}} \Psi_{ \pm}\left(\boldsymbol{k}, \boldsymbol{k}_{2}, \ldots, \boldsymbol{k}_{n}\right) \\
a_{ \pm}^{*}(\boldsymbol{k}): \Psi\left(\boldsymbol{k}_{1}, \ldots, \boldsymbol{k}_{n}\right) \mapsto \sqrt{\frac{2 \omega(\boldsymbol{k})}{n+1}} \sum_{i=1}^{n+1} \delta\left(\boldsymbol{k}-\boldsymbol{k}_{i}\right) \Psi_{ \pm}\left(\boldsymbol{k}_{1}, \ldots, \hat{\boldsymbol{k}}_{i}, \ldots, \boldsymbol{k}_{n+1}\right)
\end{gathered}
$$

where $\Psi_{ \pm}$is an $n$-particle vector in $\mathscr{F}_{ \pm}$.

\footnotetext{
$\star$ Research supported by the Science Research Council, London.
} 
Define a unitary representation of the restricted Poincare group, $\mathscr{P}_{+}^{\uparrow}$, on $\mathscr{F}_{ \pm}$by the usual formula;

$$
\begin{aligned}
\mathscr{P}_{+}^{\dagger} \ni\{a, \Lambda\} \mapsto U_{ \pm}(a, \Lambda), \\
U_{ \pm}(a, \Lambda): \Psi_{ \pm}\left(\boldsymbol{k}_{1}, \ldots, \boldsymbol{k}_{n}\right) \mapsto e^{i \sum_{j=1}^{n}\left(k_{j}, a\right)} \Psi_{ \pm}\left(\boldsymbol{\Lambda}^{-1} \boldsymbol{k}_{1}, \ldots, \boldsymbol{\Lambda}^{-1} \boldsymbol{k}_{n}\right) \mid k_{i}^{0}=\omega\left(\boldsymbol{k}_{i}\right) .
\end{aligned}
$$

Putting $U(a, \Lambda)=U_{+}(a, \Lambda) \otimes U_{-}(a, \Lambda)$, we obtain a unitary representation of $\mathscr{P}_{+}^{\uparrow}$ in $\mathscr{F}$ such that

$$
U(a, \Lambda) \phi(x) U(a, \Lambda)^{-1}=\phi(\Lambda x+a) .
$$

\section{The Local Field Algebras}

It is standard that $\phi(f)+\phi^{*}(f), i \phi(f)-i \phi^{*}(f)$ are essentially selfadjoint on the set of finite-particle vectors in $\mathscr{F}(f$ is a real test function, in $\mathscr{D}\left(\mathbb{R}^{4}\right)$, say).

Definition 1. Let $\mathcal{O} \subset \mathbb{R}^{4}$ be a bounded open set. $\mathfrak{F}_{1}(\mathcal{O})$ is the von Neumann algebra generated by the spectral resolutions of the self-adjoint closures of $\phi(f)+\phi^{*}(f)$ and $i \phi(f)-i \phi^{*}(f)$, as $f$ runs over $\mathscr{D}(\mathcal{O})$.

Definition 2. $\mathfrak{F}_{2}(\mathcal{O})$ is the von Neumann algebra generated by the spectral resolutions of the self-adjoint closures of $\phi_{+}(f) \otimes \mathbf{1}$ and $\mathbf{1} \otimes \phi_{-}(f)$ as $f$ runs over $\mathscr{D}(\mathcal{O})$; where $\phi_{ \pm}(x)$ is the usual hermitian relativistic field in $\mathscr{F}_{ \pm}$.

Evidently $\left\{\mathfrak{F}_{1}(\mathcal{O}) \mid \mathcal{O} \subset \mathbb{R}^{4}\right\}$ and $\left\{\mathfrak{F}_{2}(\mathcal{O}) \mid \mathcal{O} \subset \mathbb{R}^{4}\right\}$ are both relativistically covariant theories in that

$$
U(a, \Lambda) \mathfrak{F}_{i}(\mathcal{O}) U(a, \Lambda)^{-1}=\mathfrak{F}_{i}(\Lambda \mathcal{O}+a)
$$

defines a representation of $\mathscr{P}_{+}^{\uparrow}$ by automorphisms of $\mathfrak{F}_{i}=\overline{\overline{\bigcup_{\mathcal{O}} \mathfrak{F}_{i}(\mathcal{O})}} \quad i=1,2$. (The double bar denotes the operator norm closure.)

Suppose $f \in \mathscr{D}(\mathcal{O})$. Then $\phi_{+}(f) \otimes 1$ is affiliated to $\mathfrak{F}_{2}(\mathcal{O})$. However, a straightforward calculation yields

$$
\phi_{+}(f) \otimes \mathbf{1}=\frac{1}{2}\left[\phi(f)+\phi^{*}(f)-i\left(\pi(g)-\pi^{*}(g)\right)\right]
$$

where $\tilde{g}(k)=\tilde{f}(k) \omega^{-1}(\boldsymbol{k})$, with $\tilde{f}(k)=(2 \pi)^{-2} \int f(x) e^{i(k, x)} d^{4} x$.

Now $f$ and $g$ are antilocal with respect to each other; at equal time $t$, say, they can only both vanish in an open set in $\mathbb{R}^{3}$ if they both vanish identically as functions of $\boldsymbol{x}$ for this time $t$ [1].

We see, therefore, that $(*)$ is associated with a bounded region in the $\mathfrak{F}_{2}$-description, but is "spread out" in the $\mathfrak{F}_{1}$-description. That is, there 
are elements in each $\mathfrak{F}_{2}(\mathcal{O})$ that cannot be accommodated in any $\mathfrak{F}_{1}\left(\mathcal{O}^{\prime}\right)$ for some bounded open set $\mathcal{O}^{\prime}$; and vice versa.

The $\mathfrak{F}_{1}$-description is preferable since it associates the charged field $\phi(f)$ with the region $\mathcal{O}$ whenever $f$ has support in $\mathcal{O}$.

\section{References}

1. Segal,I.E., Goodman,R.W.: Anti-Locality of Certain Lorentz-Invariant Operators. J. Math. Mech. 14, 629-638 (1965).

2. Borchers, H.J.: Über die Mannigfaltigkeit der interpolierenden Felder zu einer kausalen $S$-Matrix. Nuovo Cimento 15, 784-794 (1960).

I. F. Wilde

Seminar für Theoretische Physik, ETH, Hönggerberg,

CH-8049 Zürich, Switzerland 